\title{
La Jornada Universitaria de Desarrollo Científico en la UNAN-Managua
}

\section{The University Journey on Scientific Development at the UNAN- Managua}

DOI: http://dx.doi.org/10.5377/uyc.v10i16.6137

Concepción de María Mendieta. connymendieta@yahoo.com Universidad Nacional Autónoma de Nicaragua, Managua.

(C) UNAN-Managua

Recibido: octubre 2017. Aprobado: octubre 2017

\section{RESUMEN}

La Jornada Universitaria de Desarrollo Científico (JUDC) nace como un movimiento en los años 80 con la finalidad de crear iniciativas técnicas, académicas y metodológicas en docentes y estudiantes universitarios como una forma de contrarrestar el bloqueo económico impuesto por los Estados Unidos de Norteamérica a Nicaragua (Aburto Jarquin, 2015). Es por tanto un “...legado cultural de la Revolución Popular Sandinista, en el contexto de la implantación y siembra de la Investigación Científica en el complejo funcional universitario..." (De Castilla, 2012, párr.2).

Palabras clave: investigación, ciencia, estudiantes, universidad.

\begin{abstract}
SUMMARY
The University Journey on Scientific Development (JUDC) was born as a movement in the 80 s with the purpose of creating technical, academic and methodological initiatives in teachers and university students as a way to counteract the economic blockade imposed by the United States of America to Nicaragua (Aburto Jarquin, 2015). It is therefore a "... cultural legacy of the Sandinista Popular Revolution, in the context of the implantation and sowing of Scientific Research in the university functional complex ..." (De Castilla, 2012, para.2).
\end{abstract}

Keywords: research, science, students, university.

La Jornada Universitaria de Desarrollo Científico (JUDC) nace como un movimiento en los años 80 con la finalidad de crear iniciativas técnicas, académicas y metodológicas en docentes y estudiantes universitarios como una forma de contrarrestar el bloqueo económico impuesto por los Estados Unidos de Norteamérica a Nicaragua (Aburto Jarquin, 2015). Es por tanto un “...legado cultural de la Revolución Popular Sandinista, en el contexto de la implantación y siembra de la Investigación Científica en el complejo funcional universitario..." (De Castilla, 2012, párr.2). 
En 1982, la JUDC se institucionaliza en la UNAN-Managua, convirtiéndose en un espacio académico/estudiantil. Desde ese momento la JUDC logra posicionarse como una actividad macro en la planificación anual de la función investigación. Hasta la fecha se ha desarrollado por 35 años de manera ininterrumpida.

Este evento académico se realiza en cada Facultad y en el Instituto Politécnico de la Salud (IPS), coordinado por la Comisión Central de Investigación de la Universidad. Cada JUDC facultativa se logra gracias a la actividad investigativa que docentes y estudiantes realizan durante el proceso de enseñanzaaprendizaje. Cada año docentes y estudiantes se unen motivados por la necesidad de desarrollar competencias investigativas, innovadoras y emprendedoras.

Según registros correspondientes a la JUDC, desde el año 2011 al año 2016, el número de trabajos de investigación científica, ha crecido considerablemente al igual que el número de estudiantes expositores y docentes tutores. Esto indica que hay un masivo interés por la investigación, cada vez más sentida y atinada al contexto social, económico, científico y tecnológico de nuestro país, gracias a un proceso de actualización constante de las líneas de investigación de la UNAN-Managua. En la figura 1, se observa el crecimiento anual de la JUDC tomado tres aspectos elementales: cantidad de trabajos presentados, estudiantes expositores y tutores.

Otro aspecto fundamental de la JUDC es la variedad de trabajos de investigación científica que presentan los estudiantes proyectistas, desde protocolos de investigación hasta monografías, precisamente este nivel de alcance y flexibilidad, hacen de esta jornada un proceso inclusivo donde participan estudiantes desde los primeros hasta los últimos años de todas las carreras de la UNANManagua, lo cual se vincula con el Modelo Educativo de la UNAN-Managua, estrictamente derivado de la misión y visión de esta universidad.

De cara a la celebración de la XXXVI Jornada de Desarrollo Científico, año 2017, con el lema “De la Innovación al Emprendimiento" se espera una mayor cantidad de estudiantes proyectistas, así como de docentes tutores y en consecuencia trabajos de investigación. En la tabla 1, se presenta la organización por fechas, de la JUDC por facultad.

De esta manera la UNAN-Managua, continúa en su afán por la mejora continua a través de la investigación y su vinculación con el quehacer universitario. 


\section{universidâd}



Figura 1. Tendencia de la JUDC año 2011-2016. (Fuente: UNAN-Managua, 2017).

Tabla 1. Desarrollo de la XXXVI JUDC Facultativas, año 2017

\begin{tabular}{|l|c|}
\hline \multicolumn{1}{|c|}{ Facultad } & Fechas de realización \\
\hline Humanidades y Ciencias Jurídicas & Del 1 al 30 de septiembre \\
\hline Facultad de Educación e Idiomas & 8 y 9 de septiembre \\
\hline FAREM-Matagalpa & 21 y 22 de septiembre \\
\hline FAREM-Estelí & del 26 al 30 de septiembre \\
\hline FAREM-Chontales & 29 y 30 de septiembre \\
\hline Instituto Politécnico de la Salud & 28 y 29 de septiembre \\
\hline Facultad de Ciencias Económicas & del 16 al 20 de octubre \\
\hline FAREM-Carazo & 26 y 27 de octubre \\
\hline Facultad de Medicina & Del 23 al 28 de octubre \\
\hline Facultad de Ciencias e Ingeniería & 25 y 26 de octubre \\
\hline
\end{tabular}

\section{REFERENCIAS}

Aburto Jarquin, P. (2015). Indicadores de Costo de la JUDC. Un avance hacia el camino de la calidad en la UNAN-MANAGUA. (en imprenta)

De Castilla, M. (Octubre de 2012). Octubre en la Universidad: las JUDC. El Nuevo Diario, sección Opinión. Recuperado de: http://www.elnuevodiario.com.ni/opinion/267431-octubreuniversidad-judc/

Universidad Nacional Autónoma de Nicaragua, Managua. (2017). Evaluacion de Impacto de la JUDC en la UNAN-Managua, Periodo 2011-2016. (investigación en proceso). 